\title{
A Low-fat Diet Decreases High Density Lipoprotein (HDL) Cholesterol Levels by Decreasing HDL Apolipoprotein Transport Rates
}

Eliot A. Brinton, Shlomo Eisenberg," and Jan L. Breslow

Laboratory of Biochemical Genetics and Metabolism, The Rockefeller University, New York, New York 10021; and

*Department of Medicine B, Hadassah University Hospital, Jerusalem, Israel

\section{Abstract}

Diets that reduce atherosclerosis risk lower levels of HDL cholesterol (HDL-C), but the significance of this is unclear. To better understand the mechanism of this phenomenon we studied the turnover of HDL apolipoproteins A-I and A-II in 13 subjects on two contrasting metabolic diets. Upon changing from high to low intake of saturated fat and cholesterol the mean HDL-C decreased $29 \%$ from $56 \pm 13$ (SD) to $40 \pm 10$ $\mathrm{mg} / \mathrm{dl}$, while apo A-I levels fell $23 \%$ from $139 \pm 22$ to $107 \pm 22$ $\mathrm{mg} / \mathrm{dl}$ (both $P<0.001$ ). Mean apo A-II levels did not change. The fractional catabolic rate (FCR) of apo A-I increased $11 \%$ from $0.228 \pm 0.048$ to $0.254 \pm 0.063$ pools/d, while its absolute transport rate (TR) decreased $14 \%$ from $12.0 \pm 2.7$ to $10.3 \pm 3.4$ $\mathrm{mg} / \mathrm{kg}$ per d (both $P=0.005)$. The decrease in HDL-C and apo A-I levels correlated with the decrease in apo A-I TR $(r=0.79$ and 0.83 , respectively; $P<0.001$ ), but not with the increase in apo A-I FCR ( $r=-0.04$ and -0.02 , respectively). In contrast, within each diet the HDL-C and apo A-I levels were inversely correlated with apo A-I FCR both on the high-fat $(r=-0.85$ and $-0.77, P<0.001$ and $=0.002$, respectively) and low-fat diets $(r=-0.67$ and $-0.48, P=0.012$ and 0.098 , respectively) but not with apo A-I TR.

In summary, diet-induced changes in HDL-C levels correlate with and may result from changes in apo A-I TR. In contrast, differences in HDL-C levels between people on a given diet correlate with and may result from differences in apo A-I FCR. Therefore, the mechanism of dietary effects on HDL levels differs substantially from the mechanism explaining the differences in levels between individuals on a fixed diet. In assessing coronary heart disease risk, it may be inappropriate to conclude that diet-induced decreases in HDL are equivalent to low HDL within a given diet. (J. Clin. Invest. 1990. 85:144-151.) apo AI • apo AII • turnover • high density lipoprotein $\bullet$ high-fat diet $\bullet$ low-fat diet

\section{Introduction}

Population studies suggest that HDL cholesterol (HDL-C) ${ }^{1}$ levels are a potent protective risk factor against coronary heart disease (1). Heightened interest in HDL has resulted in many

Address correspondence to Dr. Eliot A. Brinton, The Rockefeller University, 1230 York Avenue, New York, NY 10021.

Received for publication 10 May 1989 and in revised form 19 September 1989.

1. Abbreviations used in this paper: FCR, fractional catabolic rate; HDL-C, HDL cholesterol; HL, hepatic lipase; LDL-C, LDL cholesterol; LPL, lipoprotein lipase; $M$, monounsaturated; $P$, polyunsaturated; S, saturated; TG, triglyceride; TR, transport rate; VLDL-C, VLDL cholesterol.

J. Clin. Invest.

(c) The American Society for Clinical Investigation, Inc.

0021-9738/90/01/0144/08 $\$ 2.00$

Volume 85, January 1990, 144-151 studies on the effects of diet on HDL-C and HDL apolipoprotein levels. Both the amount (2-4) and composition of fat (2, $5)$ as well as the amount of cholesterol $(2,5,6)$ in the diet may alter HDL-C levels in human subjects. Paradoxically, those diets most effective in lowering the harmful LDL cholesterol (LDL-C) levels usually cause the greatest decrease in the protective HDL-C levels $(2,5,7)$, leading some to question the benefits of these diets $(8,9)$. Despite the need to better understand the mechanism by which diet affects HDL-C levels, there have been only three published turnover studies in this regard in human subjects. Two of these used a paired study design. Blum et al. (10) studied the effect of changing from a high-fat to an extremely low-fat liquid formula diet. The three female subjects who adhered to the diet had a $38 \%$ decrease in HDL-C levels, a $48 \%$ increase in total HDL apolipoprotein fractional catabolic rate (FCR), and no change in transport rate (TR). Shepherd et al. (11) studied the effect of changing the type of fat from a polyunsaturated/saturated (P/S) ratio of 0.25 to 4 at a fixed $40 \%$ fat intake in four male subjects. A $33 \%$ mean decrease in HDL-C levels was associated with a $26 \%$ decrease in the TR of apo A-I with no change in its FCR. The third study, by Nestel et al. (12), compared a group of seven vegetarians (26\% fat intake, P/S 1.6 , cholesterol $<100 \mathrm{mg} / \mathrm{d})$ with a group of six control subjects on a typical Western diet (36-43\% fat intake, $P / S 0.2$ to 0.4 , cholesterol $500-700 \mathrm{mg} / \mathrm{d}$ ). The vegetarians had $9 \%$ lower HDL-C than the controls and a 59\% higher apo A-I FCR, while the apo A-I TR did not differ. Unfortunately, interpretation of these three studies is limited by small numbers of subjects, artificial dietary conditions, and/or unpaired study design.

To address the mechanism whereby antiatherogenic dietary therapy lowers HDL-C levels, we have studied the turnover of the major HDL apolipoproteins, apo A-I and apo A-II, in a group of 13 subjects consuming two contrasting diets, one high in total and saturated fat and cholesterol and the other very low in these constituents. This intervention decreased mean HDL-C and apo A-I levels but did not change mean apo A-II levels. The amount of change in HDL-C and apo A-I levels varied among the subjects and correlated with the change in apo A-I TR but not apo A-I FCR. Thus, dietary alterations in apo A-I TR best account for the effects of this dietary change on HDL-C levels.

\section{Methods}

Subjects. Five male and eight female subjects were recruited from three sources: (a) patients from the clinic of the Laboratory of Biochemical Genetics and Metabolism; $(b)$ healthy, adult volunteers working on the staff at Rockefeller University or at neighboring institutions; and (c) undergraduate students on a work-study program. The subjects were selected for fasting HDL-C levels $>30 \mathrm{mg} / \mathrm{dl}$ and triglyceride (TG) levels below the 90th percentile for age and sex (13) while on the high-fat study diet. All subjects were free from hepatic, renal, thyroid, and immunologic disorders by history and laboratory screening. Seven of the eight female subjects in this study were part of a recent report from this laboratory (14) in which the results from the high-fat diet 
were used in a study of the metabolic basis of elevated HDL-C levels. The eighth woman, subject 3 , was taking the combination contraceptive Trinorinyl $(0.035 \mathrm{mg}$ ethinyl estradiol days $1-21,0.5 \mathrm{mg}$ norethindrone days 1-7 and 17-21, and $1 \mathrm{mg}$ days 8-16 of each cycle; Syntex Laboratories, Inc., Palo Alto, CA) in a 28-d cycle. Both studies were performed on the medication with the tracer injection given on day 4 of the cycle for the high-fat diet and day 5 for the low-fat diet. Excluding this subject did not appreciably alter either the paired $t$ test or linear regression analysis test. None of the other subjects was taking medications known to alter lipid levels. The clinical aspects of these studies were approved by the Institutional Review Board of the Rockefeller University, and informed consent was obtained from the subjects in all cases.

Apolipoprotein preparation and labeling. Apo A-I and A-II were prepared from healthy donor plasma by ultracentrifugation, delipidation, and column chromatography using standard methods as previously described (14). The purified apo A-I and apo A-II were radioiodinated by the iodine monochloride method and tested for pyrogenicity and sterility as outlined recently (14).

Kinetic studies. The subjects were admitted to the Rockefeller University Hospital inpatient ward and kept on two natural-food metabolic diets for 4 wk each (Table I). The high-fat diet had a caloric distribution of $42 \%$ fat $(24 \% \mathrm{~S}, 16 \%$ monounsaturated [M], and $2 \% \mathrm{P}$; $\mathrm{P} / \mathrm{S}=0.1), 43 \%$ carbohydrate, and $15 \%$ protein with $215 \mathrm{mg}$ cholesterol per 1,000 calories. The fat content corresponded to the 50th percentile of previous American intake according to the Lipid Research Clinic (LRC) study (15), and to the 75th percentile of the current American consumption according to the National Health and Nutrition Examination II survey (16). In contrast, the low-fat diet consisted of $9 \%$ fat $(2 \% \mathrm{~S}, 4 \% \mathrm{M}, 2 \% \mathrm{P}, \mathrm{P} / \mathrm{S}=1), 76 \%$ carbohydrate, and $15 \%$ protein with $40 \mathrm{mg}$ cholesterol per 1,000 calories. This diet is comparable in fat content and $\mathrm{P} / \mathrm{S}$ ratio to rural Japanese diets in the 1960 s and 1970 s $(17,18)$. The two diets were fed in a variable order, usually with 2 or more wk intervening in addition to the 2 wk of dietary equilibration before the turnover study itself. Caloric need was estimated by the Harris-Benedict (19) equation with adjustment for physical activity (20), and caloric intake was not altered during the study. After the first $2 \mathrm{~d}$ body weight remained constant. No alcohol intake was allowed and subjects were instructed to maintain their usual level of physical activity.

One subject (No. 11) was not on the metabolic diet during his low-fat study. However, before the study he was instructed extensively by a staff dietitian regarding the appropriate diet and the careful keeping of food records. A meticulous record of all food intake kept by the patient throughout the turnover study was reviewed afterward by the dietitian with the patient and then analyzed using the same food tables on which the metabolic diet was based. Since it was determined that his intake did not differ significantly from the low-fat metabolic diet used in the other subjects, and since his weight, lipoprotein, and apolipoprotein values were constant during the turnover period, his low-fat diet results were included in the analysis.

After 2 wk of equilibration on the metabolic diet the subjects received 10 or $25 \mu \mathrm{Ci}$ each of radioiodinated apo A-I and A-II ( ${ }^{125} \mathrm{I}$ and

Table I. Summary of Metabolic Diet Composition as Percent of Total Calories (except Cholesterol)

\begin{tabular}{lrrrrrrr}
\hline & \multicolumn{7}{c}{ Fat } \\
\cline { 2 - 5 } Diet & \multicolumn{1}{c}{$\mathrm{S}$} & $\mathrm{M}$ & $\mathrm{P}$ & Total & Carbohydrate & Protein & Cholesterol \\
\hline & & & & & & & $\mathrm{mg} / 1,000 \mathrm{cal}$ \\
High fat & $23.6 \%$ & $16.5 \%$ & $1.8 \%$ & $41.9 \%$ & $42.6 \%$ & $15.5 \%$ & 215 \\
Low fat & $2.1 \%$ & $4.2 \%$ & $2.3 \%$ & $8.6 \%$ & $75.5 \%$ & $16.0 \%$ & 40 \\
& & & & & & & \\
\hline
\end{tabular}

Values are based on the United States Department of Agriculture Eighth Handbook of Food Composition (and corresponding series).
${ }^{131}$ I, respectively, or vice versa) by intravenous bolus injection. Previous studies in two separate laboratories demonstrated that radiolabeling of purified HDL apolipoprotein gives results similar to that obtained by protein labeling of intact $\operatorname{HDL}(21,22)$. This similarity is due to the rapid association of free HDL apolipoprotein with plasma HDL in vivo. To allow for thorough mixing of the tracers with HDL in the plasma compartment, the first blood sample was taken after 10 min. Blood was then taken at $4,12,24$, and $36 \mathrm{~h}$, and then daily until $14 \mathrm{~d}$. All except the 4, 12-, and 36-h samples were taken after an overnight fast of $12 \mathrm{~h}$. These samples were handled and counted as stated in our previous report (14). Since all subjects were in steady state with regard to apo A-I and A-II levels, the radioactive decay curve was plotted directly from the die-away of plasma counts. The FCR was calculated from this curve by the Matthews method (23). The absolute TR was calculated by multiplying the FCR by the plasma pool (apolipoprotein level times plasma volume) and dividing by the body weight. Alternate terms for TR include synthetic rate, production rate, or flux. All four have been used interchangeably, although synthetic rate and production rate have been the standard terms in the HDL turnover literature. Nevertheless, to make clear that appearance of apolipoprotein in the plasma was being measured, rather than the actual intracellular synthesis of protein, TR has been chosen for this report. Plasma volume was determined by isotope dilution at the 10 -min point.

To show that the iodinated apo A-I and A-II associated with HDL in vivo, in each study extra aliquots of plasma were taken $10 \mathrm{~min}$ and 7 and $14 \mathrm{~d}$ after injection. Three fractions were prepared by ultracentrifugation, $d<1.063,1.063<d<1.21$, and $d>1.21$. The density distribution of radiolabel was calculated as a percentage of total counts, and the results for the 10-min and 7- and 14-d samples were averaged. On the high-fat diet these density fractions contained $1.3,88.1$, and $10.7 \%$ of the apo A-I and 1.0, 91.2, and $8.0 \%$ of the apo A-II label, respectively.

Lipid, lipoprotein, and apolipoprotein determinations. On days 1 , $3,7,10$, and 14 of the turnover period plasma was obtained after a 12-h overnight fast for measurement of lipid, lipoprotein, and apolipoprotein levels. Lipid and lipoprotein measurements were done on fresh specimens by enzymatic methods as previously described (14). Aliquots of plasma were also stored at $-70^{\circ} \mathrm{C}$ for subsequent apo determinations. Apo A-I levels were measured by a sandwich ELISA using a polyclonal goat antibody to human apo A-I (generously supplied by Dr. Peter Herbert, The Miriam Hospital, Brown University) as recently reported (14). Apo A-II levels were kindly determined by Dr. John J. Albers in the Northwest LRC laboratories by an RIA based on a radial immunodiffusion assay (24). Over the 2-wk turnover period no temporal trends were observed in the lipid, lipoprotein, and apolipoprotein levels, and the mean of all five determinations was used in the data analysis.

Postheparin lipase activities. After $11 \mathrm{~d}$ on the test diet and $3 \mathrm{~d}$ before isotope injection, a postheparin lipase test was performed. Blood was drawn $15 \mathrm{~min}$ after an intravenous bolus dose of heparin, 60 $\mathrm{U} / \mathrm{kg}$ body weight, and the plasma was stored at $-70^{\circ} \mathrm{C}$ until assay. Lipase activity was measured with $\left[{ }^{3} \mathrm{H}\right]$ triolein in a sonicated Triton $\mathrm{X}-100$ emulsion in the presence or absence of rabbit anti-human lipoprotein lipase (LPL) serum as reported previously (14). Lipase activity was expressed as micromoles FFA liberated per milliliter plasma per hour. LPL activity was the difference between total activity and that remaining after antibody inhibition, whereas the latter was the hepatic lipase (HL) activity.

Statistical analysis. Linear regression analysis was performed using the least squares method, and statistical significance was defined as a $P$ $<0.05$. Paired and unpaired comparisons were made using the $t$ test and the same $P$ value cutoff. Calculations were performed on The Rockefeller University Hospital CLINFO system.

\section{Results}

Demographic and lipoprotein data on each subject are given in Table II. The body mass index was calculated using the mean 


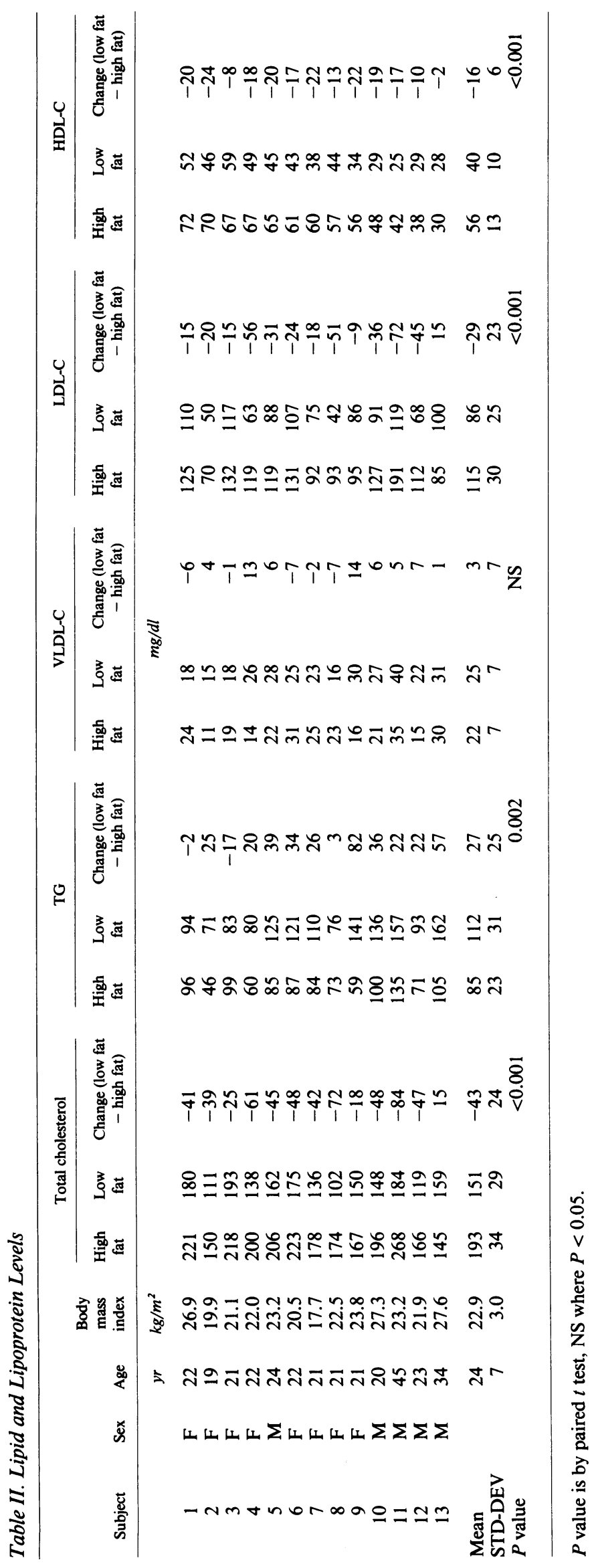

daily weight during the high-fat diet period. Mean weight on the low-fat diet did not differ significantly (group average 65.6 $\mathrm{kg}$ on the high-fat diet and $66.1 \mathrm{~kg}$ on the low-fat diet, $P>0.1$, range of differences 2.73 to $-2.05 \mathrm{~kg}$ ). The mean lipid and lipoprotein levels on each diet and the change between low-fat and high-fat diets are given. All except the VLDL cholesterol (VLDL-C) levels changed significantly. Mean total cholesterol decreased $22 \%$ from $193 \pm 34$ (SD) to $151 \pm 29 \mathrm{mg} / \mathrm{dl}$, mean LDL-C decreased $25 \%$ from $115 \pm 30$ to $86 \pm 25 \mathrm{mg} / \mathrm{dl}$, and mean HDL-C decreased $29 \%$ from $56 \pm 13$ to $40 \pm 10 \mathrm{mg} / \mathrm{dl}$ (all $P<0.001)$. TG, in contrast, increased $32 \%$ from $85 \pm 23$ to $112 \pm 31 \mathrm{mg} / \mathrm{dl}(P=0.002)$.

The levels and metabolic parameters of apo A-I and A-II for each subject are presented in Table III. Plasma apo A-I decreased in all subjects by a mean of $23 \%$, from $139 \pm 22$ to $107 \pm 22 \mathrm{mg} / \mathrm{dl}(P<0.001)$. Apo A-I FCR increased $11 \%$ from $0.228 \pm 0.048$ to $0.254 \pm 0.063$ pools/d and apo A-I TR decreased $14 \%$ from $12.0 \pm 2.7$ to $10.3 \pm 3.4 \mathrm{mg} / \mathrm{kg}$ per d (both $P$ $=0.005$ ). In contrast, there was no significant mean change in apo A-II levels. Apo A-II FCR, however, increased $6 \%$ from $0.186 \pm 0.035$ to $0.197 \pm 0.041(P=0.03)$.

Indices of HDL composition were calculated from the HDL-C, apo A-I, and apo A-II levels, and activities of LPL and HL in postheparin plasma were measured. The mean, SD, and range of these values for each diet and for the change between diets are given in Table IV. Lipid enrichment of HDL, as reflected by the HDL-C/apo A-I + apo A-II ratio, decreased $13 \%$ from $21.4 \pm 3.2$ to $18.7 \pm 4.1(P=0.009)$. The apo A-I/apo A-II ratio, related to HDL subfraction differences (25), decreased $17 \%$ from $2.86 \pm 0.58$ to $2.37 \pm 0.61(P=0.03)$. LPL activity decreased $23 \%$ from $14.9 \pm 4.5$ to $11.5 \pm 3.1 \mu \mathrm{mol} / \mathrm{ml}$ per $\mathrm{h}(P=0.05)$. HL activity decreased $17 \%$ from $13.5 \pm 6.6$ to $11.2 \pm 5.0 \mu \mathrm{mol} / \mathrm{ml}$ per h $(P=0.02)$.

Given the wide spectrum of values within each diet and of responses to dietary change, we explored interrelationships among the various study parameters by linear regression analysis. Between diets the change in HDL-C correlated with the change in apo A-I TR $(r=0.79, P=0.001)$, but not with the change in apo A-I FCR $(r=-0.04$, Fig. 1). In sharp contrast, within either the high- or low-fat diets HDL-C levels did not correlate with apo A-I TR $(r=0.21$ and 0.32 , respectively, $P$ $>0.1)$, but did correlate with apo A-I FCR $(r=-0.85$ and $-0.67, P<0.001$ and 0.01 , respectively). A somewhat different relationship was found between HDL-C and the apo A-II metabolic parameters (Fig. 2). Between diets the change in HDL-C failed to correlate significantly with the change in apo A-II TR $(r=0.34, P>0.1)$ or apo A-II FCR $(r=-0.35, P$ $>0.1$ ). However, within both high- and low-fat diets HDL-C correlated inversely with apo A-II FCR ( $r=-0.87$ and -0.81 , respectively; $P<0.001$ ). As with apo A-I, there was no correlation within diets between HDL-C and apo A-II TR ( $r$ $=-0.07$ and 0.10 for high and low fat intake, respectively).

The relationship of the apolipoprotein levels to their respective metabolic parameters was explored next. The correlations between the levels of apo A-I and its TR and FCR (Fig. 3) were similar to those of HDL-C (see Fig. 1). The changes in apo A-I levels correlated with changes in its TR $(r=0.83, P$ $<0.001$ ). However, within diets apo A-I levels and TR failed to correlate significantly on high or low fat intake $(r=0.27$ and 0.45 , respectively; $P>0.1$ ). In contrast, the change in apo A-I levels did not correlate with the change in its FCR $(r=-0.02)$, 


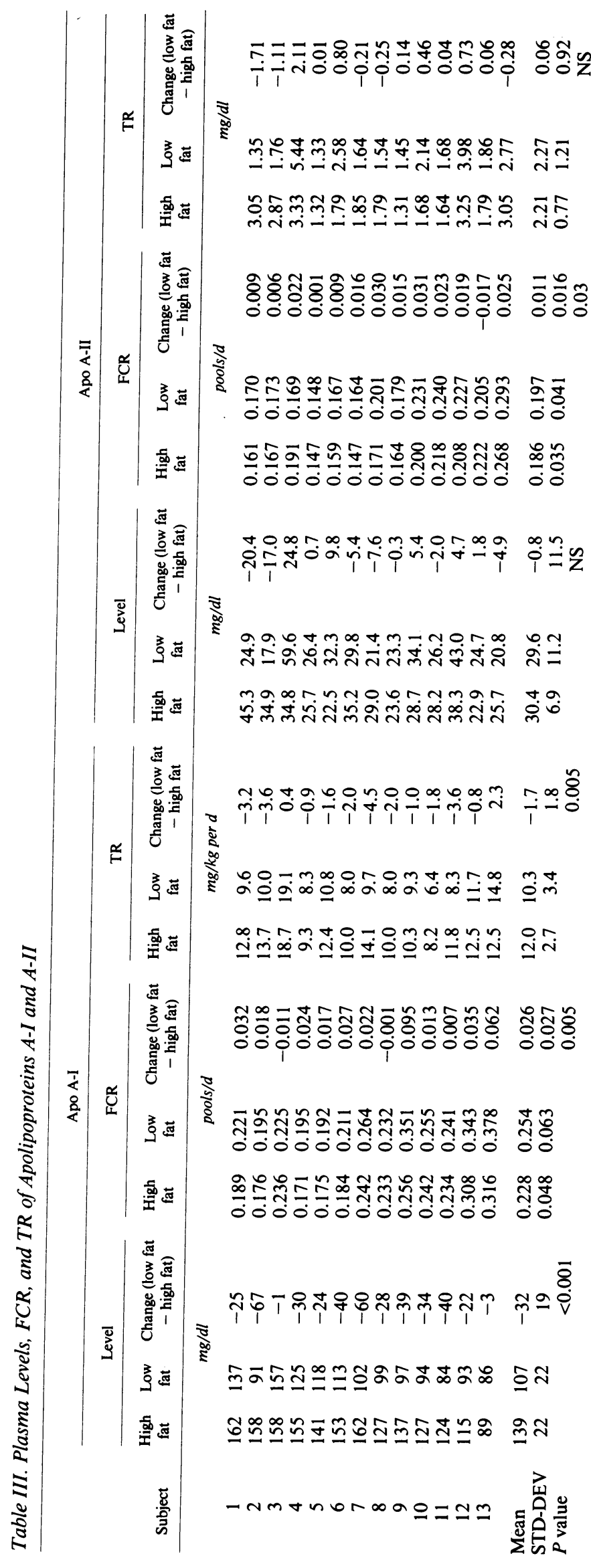

while within diets apo A-I levels and FCR correlated well on the high-fat diet $(r=-0.77, P=0.002)$, although not significantly on the low-fat diet $(r=-0.48, P=0.098)$.

Apo A-II levels related differently to the metabolic parameters than did apo A-I levels (Fig. 4). The change in levels of apo A-II correlated strongly with the change in its TR $(r=0.986, P$ $<0.001$ ), and within diets apo A-II levels also correlated with its TR $(r=0.67$ and $0.88, P=0.012$ and $<0.001$ for high- and low-fat diets, respectively). In contrast, the change in apo A-II levels did not correlate with changes in its FCR $(r=-0.40, P$ $>0.1)$, nor did they correlate within diets $(r=-0.22$ and -0.14 , high- and low-fat diets, respectively).

The relationships between HDL composition and HDL metabolism were next examined. The change in the HDL-C/ apo A-I + apo A-II and apo A-I/apo A-II ratios correlated only with the change in apo A-II TR $(r=-0.74$ and $-0.84, P$ $=0.004$ and 0.001 , respectively) and not with changes in the other metabolic parameters $(r=-0.24-0.11)$. Within diets, the inverse correlations between the HDL-C/apo A-I + apo A-II ratio and the FCRs of apo A-I and apo A-II were strong on the high-fat diet $(r=-0.70$ and $-0.77, P=0.008$ and 0.002 , respectively), but were borderline or nonsignificant on the low-fat diet $(r=-0.49$ and $-0.55, P=0.09$ and 0.05 , respectively). None of the correlations between HDL-C/apo A-I + apo A-II and the TRs of apo A-I or apo A-II on high or low fat intake reached statistical significance $(r=-0.02--0.50, P$ $>0.08$ ). The apo A-I/apo A-II ratio failed to correlate significantly with the TR or FCR of apo A-I or apo A-II on either diet, except for an inverse correlation with apo A-II TR on the high- and low-fat diets $(r=-0.75$ and $-0.73, P=0.003$ and 0.005 , respectively).

Relationships between postheparin activities of LPL and $\mathrm{HL}$ and the metabolic parameters were explored. Changes in HL correlated with changes in apo A-I TR $(r=-0.61, P$ $=0.03$ ) but not with changes in apo A-II TR or with the change in either FCR. In contrast, within diets HL correlated well with the apo A-I and apo A-II FCR on both the high-fat $(r$ $=0.81$ and 0.95 , respectively; $P<0.001)$ and low-fat diets $(r$ $=0.71$ and $0.86, P=0.01$ and $<0.001$, respectively) but not with the TR of apo A-I or apo A-II. LPL failed to correlate with any of the metabolic parameters within either diet or with changes between diets.

The inclusion of both male and female subjects allowed us to test for possible sex differences in HDL metabolism under our study conditions. Changes in the measured parameters between diets did not differ between the sexes except for the change in $\mathrm{HL}$, which decreased $4.9 \pm 2.1$ vs. $0.9 \pm 2.1 \mu \mathrm{mol} / \mathrm{ml}$ per $h$ in men and women, respectively $(P=0.01$ for the sex difference). Within diets, the men and women differed in several parameters. On high fat intake HDL-C and apo A-I levels were lower in the men than in the women $(45 \pm 13$ vs. $64 \pm 6$ and $119 \pm 19$ vs. $151 \pm 13 \mathrm{mg} / \mathrm{dl}$, respectively, $P=0.004$ ), as expected. However, among the FCRs and TRs only apo A-II FCR differed significantly $(0.215 \pm 0.039$ vs. $0.169 \pm 0.019$ pools/d for men and women, respectively $[P=0.014]$ ). Also, $\mathrm{HL}$ was $96 \%$ higher in the men $(20.0 \pm 5.7 \mathrm{vs.} 10.2 \pm 4.2 \mu \mathrm{mol} /$ $\mathrm{ml}$ per h, $P=0.006$ ). On the low-fat diet sex differences were similar to the high-fat results except for the addition of higher TG and VLDL-C levels and a lower HDL-C/apo A-I + apo A-II ratio in the men. 
Table IV. HDL Composition Indices and Postheparin Lipase Activities

\begin{tabular}{|c|c|c|c|c|c|c|}
\hline & \multicolumn{3}{|c|}{ HDL-C/apo A-I + A-II (molar ratio) } & \multicolumn{3}{|c|}{ Apo A-I/apo A-II (molar ratio) } \\
\hline & High fat & Low fat & $\begin{array}{c}\text { Change } \\
\text { (low fat - high fat) }\end{array}$ & High fat & Low fat & $\begin{array}{c}\text { Change } \\
\text { (low fat - high fat) }\end{array}$ \\
\hline Mean & 21.4 & 18.7 & -2.7 & 2.86 & 2.37 & -0.49 \\
\hline STD-DEV & 3.2 & 4.1 & 3.1 & 0.58 & 0.61 & 0.75 \\
\hline \multirow{6}{*}{$\begin{array}{l}\text { Range } \\
P \text { value }\end{array}$} & $16.1-26.4$ & $11.7-27.7$ & $-7.2-4.2$ & $2-3.8$ & $1.2-3.3$ & $-1.6-1.2$ \\
\hline & & & 0.009 & & & 0.03 \\
\hline & \multicolumn{6}{|c|}{ Postheparin lipase activity } \\
\hline & \multicolumn{3}{|c|}{ LPL } & \multicolumn{3}{|c|}{$\mathrm{HL}$} \\
\hline & High fat & Low fat & $\begin{array}{c}\text { Change } \\
\text { (low fat - high fat) }\end{array}$ & High fat & Low fat & $\begin{array}{c}\text { Change } \\
\text { (low fat }- \text { high fat) }\end{array}$ \\
\hline & \multicolumn{6}{|c|}{$\mu \mathrm{mol} / \mathrm{ml} \mathrm{per} h$} \\
\hline Mean & 14.9 & 11.5 & -3.4 & 13.5 & 11.2 & -2.3 \\
\hline STD-DEV & 4.5 & 3.1 & 5.3 & 6.6 & 5 & 2.8 \\
\hline Range & $10.4-24$ & $5.7-15.6$ & $-14-2.9$ & $5-25.4$ & $3.9-20.1$ & $-6.6-0.8$ \\
\hline$P$ value & & & 0.05 & & & 0.02 \\
\hline
\end{tabular}

\section{Discussion}

The main goals of this study were to examine the effects of clinically relevant, mixed dietary changes on the metabolism of the major HDL apolipoproteins A-I and A-II, and to explore the metabolic basis of interindividual variability in HDL response to dietary change. We used two diets at opposite ends of the usual clinical spectrum, that is, "pro" and "antiatherogenic" diets. These diets should produce the maximum clinically relevant change in HDL-C and allow study of the metabolic mechanisms of that change. While maintaining the dietary control of a metabolic ward, we mimicked an outpatient setting by using only natural, commonly available foods. Comparing the diet low in saturated and total fat and cholesterol to the one high in these factors, HDL-C and apo A-I levels fell in all subjects, although to widely variable degrees.
We also saw a rise in the mean apo A-I FCR and a fall in the apo A-I TR. Our dietary change was approximately the sum of the change in total fat intake used by Blum et al. (10) and the change in fat type studied by Shepherd et al. (11). In this light, it is interesting to consider that our metabolic findings were also a combination of the two reported findings, an increase in FCR as in the Blum study and a decrease in TR as published by Shepherd.

Our report goes beyond these two previous paired studies of diet effects on HDL metabolism. By recruiting larger numbers of subjects, with a broader range of HDL-C levels, including both men and women, we were able to explore the mechanisms of interindividual differences in diet response. Group comparisons on the high- and low-fat diets showed a signifcant rise in apo A-I FCR and fall in apo A-I TR, either of which had the potential to explain the diet-induced decreases

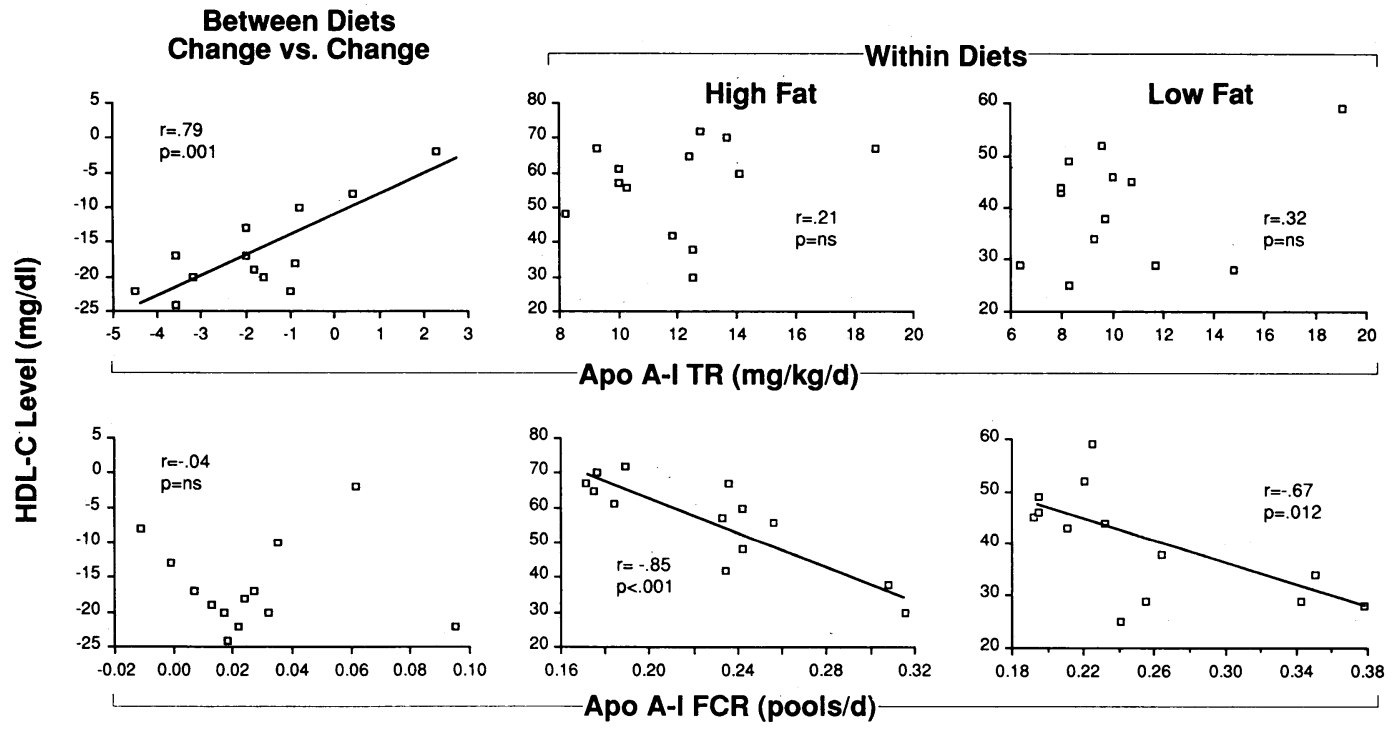

Figure 1. Relationships between levels of HDL-C $(\mathrm{mg} / \mathrm{dl})$ and apo A-I, TR ( $\mathrm{mg} / \mathrm{kg}$ per $\mathrm{d})$, and FCR (pools/d). Results are plotted as change vs. change (low-fat diet value - high-fat diet value) and as within-diet results for high and low fat intake. 


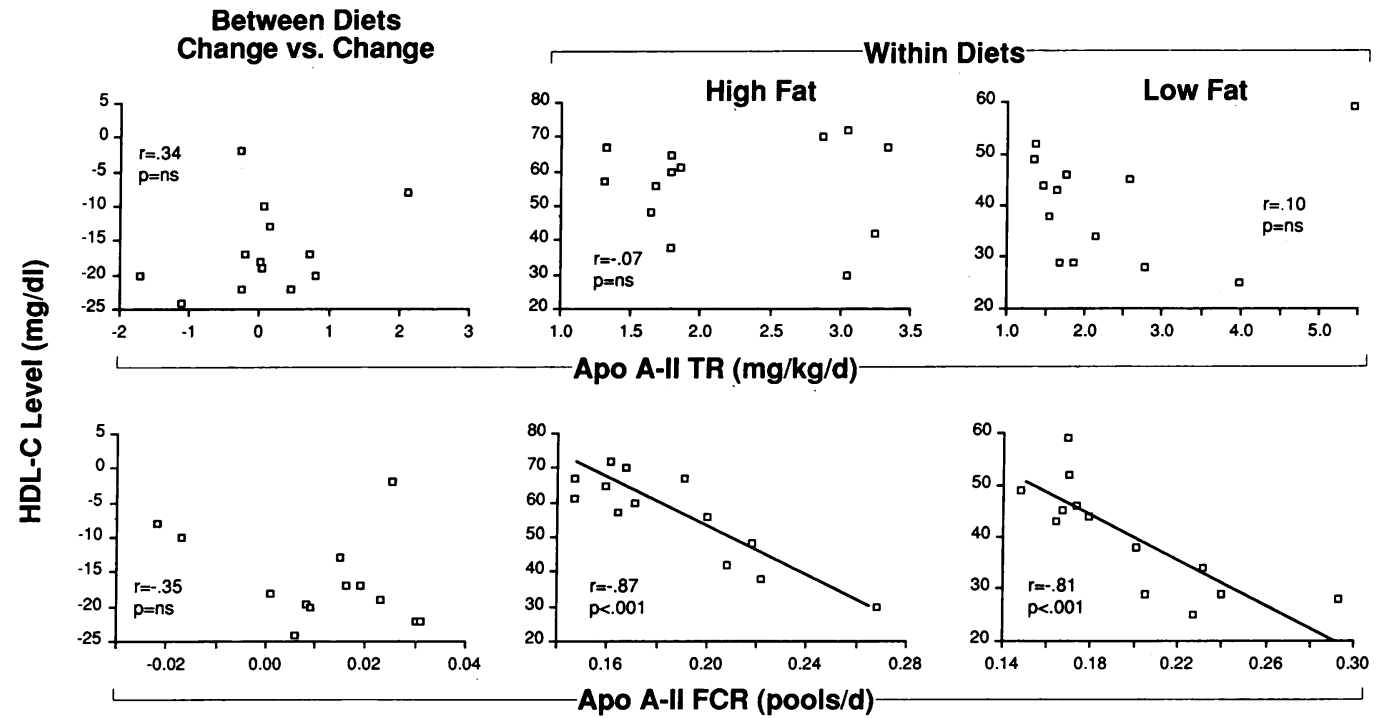

Figure 2. Relationships between levels of HDL-C $(\mathrm{mg} / \mathrm{dl})$ and apo A-II TR $(\mathrm{mg} / \mathrm{kg}$ per $\mathrm{d})$ and $\mathrm{FCR}$ (pools/d). Results are plotted as in Fig. 1. in HDL-C and apo A-I levels. However, linear regression analysis indicated that only the changes in TR correlated with the changes in these levels. This finding is particularly striking given the fact that, within both the high- and low-fat diets, HDL-C and apo A-I levels correlated only with apo A-I FCR and not with apo A-I TR, in confirmation of previous findings by ourselves (14) and others (21). Thus, we suggest that the diet-induced decreases in HDL-C and apo A-I levels may be caused by the decrease in apo A-I TR and not by the increase in FCR.

One caveat regarding our apo A-I TR data results from the way in which the TR value is obtained. As with all available turnover methods, our TR values were not measured directly, but were calculated as the FCR times the pool size, where the latter was determined using the apolipoprotein level in the mathematical equation. This opens the possibility of a statistical artefact in the correlation between the TR of an apolipoprotein and its plasma level. However, in our case the correlation between changes in apo A-I levels and TR probably was not confounded because we saw a similar correlation between changes in HDL-C levels and apo A-I TR, even though these two terms have no direct mathematical connection. Thus, the correlations of the decreases in apo A-I TR with HDL-C and apo A-I levels when changing from a high to a low fat intake appear to represent true physiological relationships.

Our results with regard to apo A-II further underscore the importance of TR in determining dietary response. Changes in apo A-II levels correlated with changes in apo A-II TR and not with changes in apo A-II FCR. Even within the high- and low-fat diets apo A-II levels correlated with apo A-II TR rather than with apo A-II FCR. Thus, despite fundamental differences between apo A-I and apo A-II in the metabolic mechanisms of their levels within a diet, changes in their levels and in HDL-C levels between diets all appear to result from changes in TR.

This finding is of particular interest in light of recent animal studies. In rabbits, Quig and Zilversmit recently reported that a sixfold increase in saturated fat intake caused a twofold increase in HDL apolipoprotein production rate (TR) and a similar increase in HDL-C and HDL apolipoprotein levels (26). Unlike our study, no change in FCR was seen, nor were there changes in HDL composition. However, it is interesting

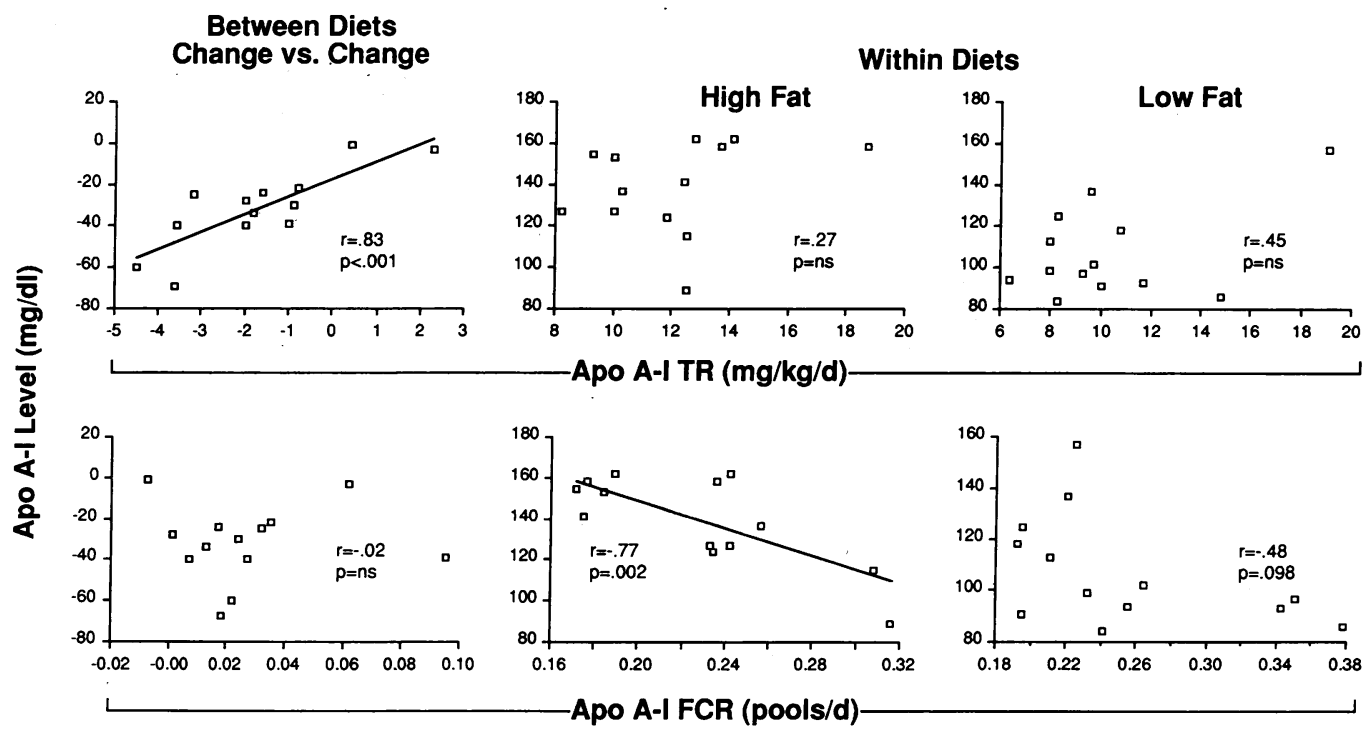

Figure 3. Relationships between levels of apo A-I $(\mathrm{mg} / \mathrm{dl})$ and its TR $(\mathrm{mg} /$ kg per d) and FCR (pools/d). Results are plotted as in Fig. 1. 


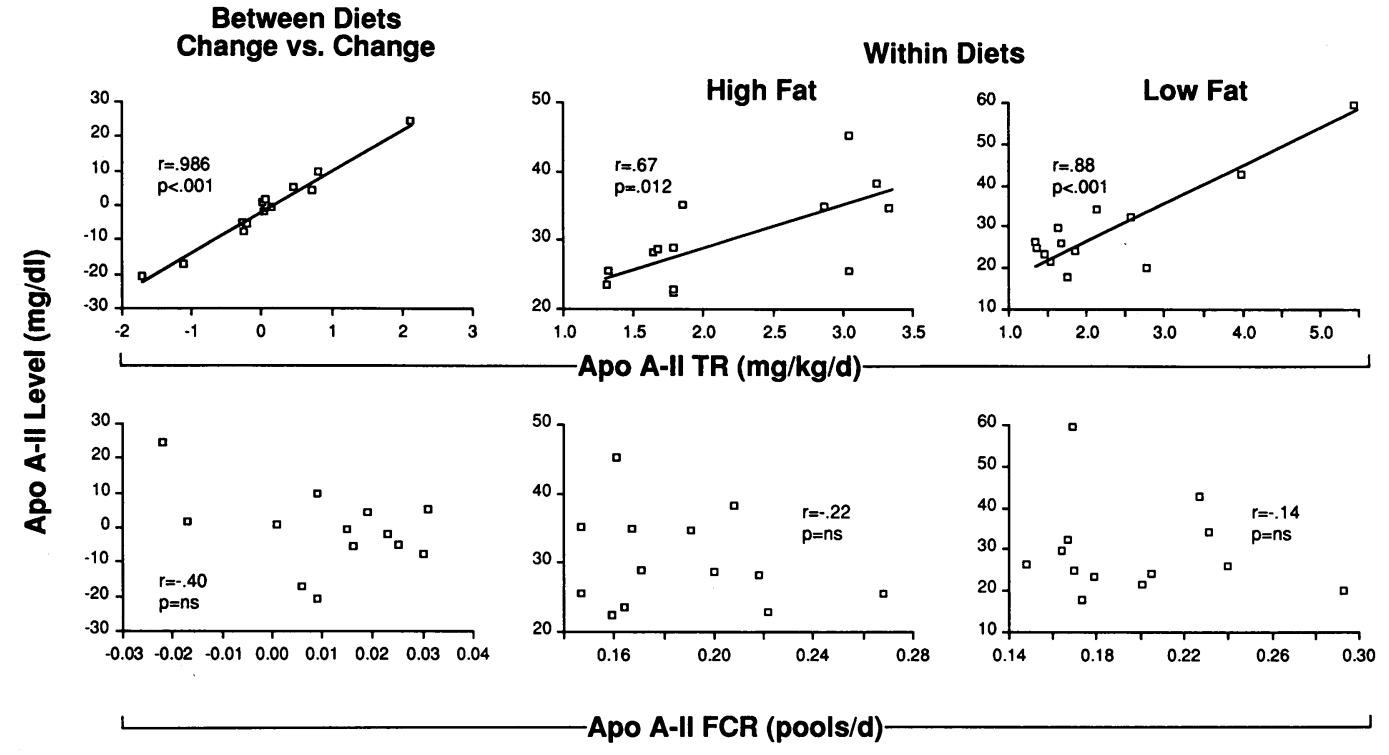

Figure 4. Relationships between levels of apo A-II $(\mathrm{mg} / \mathrm{dl})$ and its TR $(\mathrm{mg} /$ $\mathrm{kg}$ per $\mathrm{d}$ ) and FCR (pools/d). Results are plotted as in Fig. 1. that rabbits share with humans altered TR as the metabolic mechanism of dietary change in HDL-C levels. Recent work with nonhuman primates demonstrated a $16 \%$ decrease in plasma apo A-I levels upon increasing the $\mathrm{P} / \mathrm{S}$ ratio from 0.3 to 2.2 on a high-cholesterol diet (27). This was associated with a $19 \%$ decrease in hepatic synthesis of apo A-I and a $22 \%$ decrease in hepatic apo A-I mRNA, while intestinal apo A-I mRNA was unchanged. This latter study suggests that the decreased appearance of apo A-I in the plasma compartment, expressed as reduced TR, seen in the turnover studies in rabbits and humans, reflects decreased intrahepatic protein synthesis.

HL previously has been found to correlate with the FCR of apo A-I and A-II on high fat intake (28). We confirmed that finding and extended it to a low-fat diet. Thus, at either end of the usual dietary spectrum of fat and cholesterol intake, HL appears to account for a large share of intersubject variability in apo A-I FCR. This suggests that HL may play an important, if indirect, role in atherogenesis. Between diets, the change in HL did not correlate with the change in apo A-I or apo A-II FCR. However, the change in HL did correlate inversely with the change in apo A-I TR. Since the liver is both a major site of apo A-I synthesis and presumably the only source of HL, this interesting association suggests that a common intrahepatic mechanism may mediate a reciprocal response of the two factors to dietary alteration. With regard to LPL, a decrease in fat intake may decrease its activity in skeletal muscle (29) but not in adipose tissue (30). We saw a decrease in postheparin plasma LPL on decreased fat intake but it did not correlate with changes in any HDL metabolic parameter. Within diets, LPL also failed to correlate with any of the metabolic parameters.

We previously reported an inverse relationship between an index of HDL lipid enrichment, the HDL-C/apo A-I + apo A-II ratio, and the FCRs of apo A-I and A-II in 15 women on a high-fat diet (14). We felt that lipid enrichment could be either a cause or an effect of decreased FCR. That report involved seven of the eight women of this study (all except subject 3 ) but examined only the high-fat diet, a higher range of HDL-C levels, and included no men. In this study we found similar strong relationships between the HDL-C/apo A-I + apo A-II ratio and the FCRs in men and women, but only on the highfat diet. On the low-fat diet the correlations appeared weaker. This suggests that high fat intake may play a role in the relationship between HDL lipid enrichment and HDL apo FCR. A high-fat diet may enrich chylomicrons and subsequently HDL with lipids. This lipid-rich HDL may then be less susceptible to catabolism, thus lowering apolipoprotein FCR.

The question of sex differences in metabolic responses to dietary change is of interest. On both high- and low-fat diets the men had lower HDL-C and apo A-I levels and higher apo A-II FCR and HL than women. Despite these baseline differences, the only difference in diet response was in $\mathrm{HL}$, which decreased fivefold more in men than in women on going from high to low fat intake. This finding suggests that men are uniquely susceptible to induction of HL activity by high saturated fat and cholesterol intake. Elevated HL activity may have caused the higher FCRs in our male subjects and thus, perhaps, their lower HDL-C levels. Furthermore, this could be a mechanism of the elevated atherosclerosis risk seen in men in Western societies. Thus, men may have particularly compelling reasons for adherence to a prudent diet.

To summarize, we have confirmed that the major metabolic mechanism of interindividual differences in HDL-C and apo A-I levels is FCR. In contrast, we now report that the mechanism of dietary changes in these levels is not changes in FCR, but instead appears to be changes in apo A-I TR. Thus a low HDL-C on a given diet occurs by a mechanism distinct from that of a decrease in HDL-C upon changing diets. We feel that this fundamental metabolic difference renders it inappropriate to assume that a diet-induced decrease in HDL-C carries the same risk of atherosclerosis as does a low HDL-C within a given diet. This seems particularly so since epidemiologic data consistently show that populations with low-fat diets and low HDL-C have reduced atherosclerosis incidence (31-33). As a corollary, we recommend that guidelines for antiatherogenic diets continue to emphasize the lowering of LDL, rather than avoidance of HDL reduction. 


\section{Acknowledgments}

The authors are indebted to Dr. John Albers for performing the apo A-II assays, to Dr. Rudolf Zechner for establishing the lipase assay, and to Dr. Margo Denke and Dr. Li-Shin Huang for establishing the apo A-I assay. The authors thank Dr. Nassar Khan, Ms. Michelle Estilo, Ms. Margie Timmons, Ms. Katie Tsang, and Ms. Bahareh Sahami for expert technical assistance, Ms. Anne Brown for assisting with dietary design, Ms. Alexandra McNear for clerical expertise, and the turnover subjects for their patience and cooperation.

This work was supported in part by grants from the National Institutes of Health (HL-33714, HL-32435, HL-36461, and CA-29502), a Clinical Investigator Award (HL-02034), and a General Clinical Research Center grant (MO1-RR00102), in addition to a grant from the Bankers Trust Company Group, as well as a general support from the Pew Trusts. Dr. Jan L. Breslow is an Established Investigator of the American Heart Association.

\section{References}

1. Miller, G. J., and N. E. Miller. 1975. Plasma-high-density-lipoprotein concentration and development of ischaemic heart-disease. Lancet. i:16-19.

2. Schaefer, E. J., R. I. Levy, N. D. Ernst, F. D. Van Sant, and H. B. Brewer. 1981. The effects of low cholesterol, high polyunsaturated fat, and low fat diets on plasma lipid and lipoprotein cholesterol levels in normal and hypercholesterolemic subjects. Am. J. Clin. Nutr. 34:1758-1763.

3. Lewis, B., M. Katan, I. Merkx, N. E. Miller, F. Hammett, R. M. Kay, A. Nobels, and A. V. Swan. 1981. Towards an improved lipidlowering diet: additive effects of changes in nutrient intake. Lancet. ii: $1310-1313$.

4. Knuiman, J. T., C. E. West, M. B. Katan, and J. G. A. J. Hautvast. 1987. Total cholesterol and high density lipoprotein cholesterol levels in populations differing in fat and carbohydrate intake. Arteriosclerosis. 7:612-619.

5. Zanni, E. E., V. I. Zannis, C. B. Blum, P. N. Herbert, and J. L. Breslow. 1987. Effect of egg cholesterol and dietary fats on plasma lipids, lipoproteins, and apoproteins of normal women consuming natural diets. J. Lipid Res. 28:518-527.

6. Nestel, P., N. Tada, T. Billington, M. Huff, and N. Fidge. 1982. Changes in very low density lipoproteins with cholesterol loading in man. Metab. Clin. Exp. 31:398-404.

7. Ehnholm, C., J. K. Huttunen, P. Pietinen, U. Leino, M. Mutanen, E. Kostiainen, J. Pikkarainen, R. Dougherty, J. Iacono, and P. Puska. 1982. Effect of diet on serum lipoproteins in a population with a high risk of coronary heart disease. N. Engl. J. Med. 307:850-855.

8. McNamara, D. J. 1982. Diet and hyperlipidemia: a justifiable debate. Arch. Intern. Med. 142:1121-1124.

9. Mattson, F. H., and S. M. Grundy. 1985. Comparison of effects of dietary saturated, monounsaturated, and polyunsaturated fatty acids on plasma lipids and lipoproteins in man. J. Lipid Res. 26:194202.

10. Blum, C. B., R. I. Levy, S. Eisenberg, M. Hall III, R. H. Goebel, and M. Berman. 1977. High density lipoprotein metabolism in man. $J$. Clin. Invest. 60:795-807.

11. Shepherd, J., C. J. Packard, J. M. Stewart, B. D. Vallance, T. D. Lawrie, and H. Morgan. 1979. The relationship between the cholesterol content and subfraction distribution of plasma high-density lipoproteins. Clin. Chim. Acta. 101:57-62.

12. Nestel, P. J., T. Billington, and B. Smith. 1981. Low density and high density lipoprotein kinetics and sterol balance in vegetarians. Metab. Clin. Exp. 30:941-945.

13. The Lipid Research Clinics Population Studies Data Book. 1980. The Prevalence Study, Vol. I. U.S. Department of Health and Human Services, National Institutes of Health, Bethesda, MD. 52-77.

14. Brinton, E. A., S. Eisenberg, and J. L. Breslow. 1989. Elevated high density lipoprotein cholesterol levels correlate with decreased apo A-I and apo A-II fractional catabolic rate in women. J. Clin. Invest. 84:262-269.
15. The Lipid Research Clinics Population Studies Data Book. 1982. The Prevalence Study: Nutrient Intake, Vol. II. U.S. Department of Health and Human Services, National Institutes of Health, Bethesda, MD. 42.

16. National Health and Nutrition Examination Survey. 1983. Dietary intake source data. National Center for Health Statistics, Office of Health Research Statistics and Technology, Hyattsville, MD. 48-52.

17. Ueshima, H., M. Iida, T. Shimamoto, M. Konishi, M. Tanigaki, M. Doi, N. Nakanishi, Y. Takayama, H. Ozawa, and Y. Komachi. 1982. Dietary intake and serum total cholesterol level: their relationship to different lifestyles in several Japanese populations. Circulation. 66:519-526.

18. Goto, Y. 1986. Cholesterol level of Japanese in past twenty years and primary prevention study by dextran sulfate (MDS). Atherosclerosis. 7:9-13.

19. Harris, J. A., and F. G. Benedict. 1919. A biometric study of basal metabolism in man. J. B. Lippincott Co., Philadelphia.

20. Mahalko, J. R., and L. K. Johnson. 1980. Accuracy of predictions of long-term energy needs. J. Am. Diet. Assoc. 77:557-561.

21. Schaefer, E. J., L. A. Zech, L. L. Jenkins, T. J. Bronzert, E. A. Rubalcaba, F. T. Lindgren, R. L. Aamodt, and H. B. Brewer, Jr. 1982. Human apolipoprotein A-I and A-II metabolism. J. Lipid Res. 23:850-862.

22. Malmendier, C. L., C. Delcroix, and J. P. Ameryckx. 1983. In vivo metabolism of human apoprotein A-I-phospholipid complexes: comparison with human high density lipoprotein-apoprotein A-I metabolism. Clin. Chim. Acta. 131:201-210.

23. Matthews, C. M. E. 1957. The theory of tracer experiments with ${ }^{131}$ I-labelled plasma proteins. Phys. Med. Biol. 2:36-53.

24. Cheung, M. C., and J. J. Albers. 1977. The measurement of apolipoprotein A-I and A-II levels in men and women by immunoassay. J. Clin. Invest. 60:43-50.

25. Cheung, M. C., and J. J. Albers. 1979. Distribution of cholesterol and apolipoprotein A-I and A-II in human high density lipoprotein subfractions separated by $\mathrm{CsCl}$ equilibrium gradient centrifugation: evidence for HDL subpopulations with differing A-I/A-II molar ratios. J. Lipid Res. 20:200-207.

26. Quig, D. W., and D. B. Zilversmit. 1989. High density lipoprotein metabolism in a rabbit model of hyperalphalipoproteinemia. Ath erosclerosis. 76:9-19.

27. Sorci-Thomas, M., M. M. Prack, N. Dashti, F. Johnson, L. L. Rudel, and D. L. Williams. 1989. Differential effects of dietary fat on the tissue specific expression of the apolipoprotein A-I gene: relationship to plasma concentration of high density lipoproteins. J. Lipid Res. 30:1397-1403.

28. Saku, K., P. S. Gartside, B. A. Hynd, S. G. Mendoza, and M. L. Kashyap. 1985. Apolipoprotein AI and AII metabolism in patients with primary high-density lipoprotein deficiency associated with familial hypertriglyceridemia. Metab. Clin. Exp. 34:754-764.

29. Lithell, H., I. Jacobs, B. Vessby, K. Hellsing, and J. Karlsson. 1982. Decrease of lipoprotein lipase activity in skeletal muscle in man during a short-term carbohydrate-rich dietary regime: with special reference to HDL-cholesterol, apolipoprotein and insulin concentrations. Metab. Clin. Exp. 31:994-998.

30. Taskinen, M.-R., E. A. Nikkila, and A. Ollus. 1983. Serum lipids and lipoproteins in insulin-dependent diabetic subjects during high-carbohydrate, high-fiber diet. Diabetes Care. 6:224-230.

31. Connor, W. E., M. T. Cerqueira, R. W. Connor, R. B. Wallace, M. R. Malinow, and H. R. Casdorph. 1978. The plasma lipids, lipoproteins, and diet of the Tarahumara Indians of Mexico. Am. J. Clin. Nutr. 31:1131-1142.

32. Knuiman, J. T., R. J. J. Hermus, and J. G. A. J. Hautvast. 1980. Serum total and high density lipoprotein (HDL) cholesterol concentrations in rural and urban boys from 16 countries. Atherosclerosis. 36:529-537.

33. Knuiman, J. T., C. E. West, and J. Burema. 1982. Serum total and high density lipoprotein cholesterol concentrations and body mass index in adult men from thirteen countries. Am. J. Epidemiol. 116:631-642. 